\title{
REAL COMMUTATIVE SEMIGROUPS ON THE PLANE
}

\author{
J. G. HoRNe, JR.
}

A real semigroup is a topological semigroup containing a sub-semigroup $R$ isomorphic to the multiplicative semigroup of real numbers, embedded so that 1 is an identity and 0 is a zero. This paper is devoted to a preliminary study of real commutative semigroups on the plane and especially to characterizing the product semigroup on $R \times R$. It leans heavily on the fundamental paper [5] of Mostert and Shields which in turn depends the paper [1] of Faucett who, among other things, characterized the multiplicative semigroup on the closed unit interval. Characterizations of the multiplicative semigroup of nonnegative real numbers and of all real numbers were given in [4] and [3] respectively. (In connection with the latter characterization, also see [2]).

Nothing like a complete description of all real commutative semigroups on the plane can be given at this time, even under the additional hypothesis that there are no (non-zero) nilpotent elements. A crude classification can be given however on the basis of the number and arrangement of the components of the maximal subgroup $H(1)$. If $H(1)$ is connected then the semigroup is necessarily the multiplicative semigroup of complex numbers. If $H(1)$ is not connected then the component $G$ of the identity in $H(1)$ is always isomorphic to the two dimensional vector group. There can be precisely two components in $H(1)$; in this case, $H(1)$ may be dense or not. There are at least two instances of the former (see Examples 1 and 2 of $\S 6$ ) and at least two instances of the latter (see Examples 3 and 4 of $\S 6$ ). If there are more than two components, but there are no nilpotent elements, then the number of components of $H(1)$ is four and $H(1) / G$ is isomorphic to the four group. Example 5 of $\S 6$ shows that even in this case $H(1)$ need not be dense and the suggestion is that there are many instances of this case. A characterization of the product semigroup on $R \times R$ appears in $\S 5$.

The author is happy to acknowledge his indebtedness to Professor B. J. Ball for several valuable discussions concerning the topology of the plane and to Professor R. P. Hunter for calling his attention to [2].

Preliminaries. The closure of a subset $A$ of a topological space is denoted $A^{-}$. The set-theoretic difference of two sets $A$ and $B$ is denoted by $A \backslash B$.

A binary operation, or multiplication, is denoted by juxtaposition. By a semigroup $S$ we mean a topological semigroup, that is, a Hausdorff space with a continuous associative multiplication. All semigroups

Received August 15, 1960. Presented to the Society November 18, 1960. 
in this paper are assumed to be commutative, though we shall occasionally list this hypothesis for emphasis. An isomorphism between two semigroups is a function which is both an algebraic isomorphism and a homeomorphism. If there is an identity, it will be denoted by 1 . A zero will usually, though not always, be denoted by 0 .

As in [5], $H(1)$ denotes the set of elements with inverses, and $G$ denotes the component of the identity in $H(1)$. The boundary of $G$ is denoted by $L$.

By the square of an element $s$ is meant the product of $s$ with itself, and is denoted by $s^{2}$. More generally, if $n$ is a positive integer, $s^{n}$ denotes the $n$-fold product of $s$ with itself. If $s$ has an inverse it is denoted by $1 / s$. An element $e$ is an idempotent if $e^{2}=e$. In a semigroup with 0 , a nilpotent element is an element $x \neq 0$ such that $x^{n}=0$ for some positive integer $n$. (Some writers permit 0 to be a nilpotent element.)

For subsets $A, B$ of $S, A B=\{a b: a \in A, b \in B\}$. If $x \in S$ then $A x$ is the abbreviation of $A\{x\}$. An ideal is a subset $A$ such that $A S \subset A$. A sub-semigroup is a subset $A$ such that $A A \subset A$.

Throughout this work, $E$ will denote the Euclidean plane and $R$ will denote a semigroup isomorphic to the multiplicative semigroup of real numbers. The set of all positive members of $R$ is denoted by $P$ and the set of negative members by $N$.

It is a standing assumption that $E$ has the structure of a real (commutative) semigroup. That is, $E$ satisfies the condition

$\left(^{*}\right) E$ is endowed with the structure of a commutative topological semigroup which contains a sub-semigroup $R$ isomorphic to the multiplicative semigroup of real numbers. Furthermore, the elements 1 and 0 of $R$ act as an identity and a zero respectively for $E$.

We intend to imply that a fixed isomorph of the real numbers has been chosen. (In this connection see Remark 5.4). Thus -1 is a well defined element so there is no harm in abbreviating $(-1)(x)$ to $-x$. For a subset $A \subset E,-A=\{-a: a \in A\}$. The element zero is sometimes referred to as the origin.

By coordinate-wise multiplication is meant the multiplication defined on $R \times R$ by the identity: $\left(x_{1}, x_{2}\right)\left(y_{1}, y_{2}\right)=\left(x_{1} y_{1}, x_{2} y_{2}\right)$. That is, coordinatewise multiplication is simply the name of multiplication on the product semigroup $R \times R$, but this language is convenient later. Under coordinatewise multiplication, $R \times R$ satisfies condition $\left(^{*}\right)$ if $R$ is identified with the set: $\{(x, x): x \in R\}$.

1. This section is devoted to a study of the embedding and separation properties of $R$ and of certain aspects of the multiplication maps $m_{x}$, where $m_{x}$ is the map of $E$ into itself defined by the identity: $m_{x}(y)=x y$. 
1.1 Lemma. Let $u \in R$. If $x \neq 0$ and $u x=x$ then $u= \pm 1$. Therefore $m_{x}$ is a one-to-one function on $P^{-}$and on $N^{-}$which is one-to-one on $R$ if and only if $x \neq-x$.

Proof. If $u \neq \pm 1$ then either $u^{n} \rightarrow 0$ or $(1 / u)^{n} \rightarrow 0$ so $u^{n} x \rightarrow 0$ or $(1 / u)^{n} x \rightarrow 0$. However, $u^{n} x=x$ and $(1 / u)^{n} x=x$ which is a contradiction. Therefore $u= \pm 1$. The remaining conclusions now follow easily.

1.2 Theorem. For every $x, m_{x}$ is a closed map from $R$ into $E$. Hence if $x \neq 0$ then $m_{x}$ is a homeomorphism on $P^{-}$and on $N^{-}$which is a homeomorphism on $R$ if and only if $-x \neq x$, or equivalently, if and only if $R x$ cuts $E$. In any case, $R x$ is closed subset of $E$.

Proof. The assertions concerning the case $x=0$ are trivial so assume $x \neq 0$. Suppose that $t_{n}$ is a sequence in $R$ such that $t_{n} x$ converges. If $t_{n}$ does not converge then either $t_{n}$ has two cluster points $t, t^{\prime}$, or a sub-sequence $s_{n}$ of $t_{n}$ satisfies: $1 / s_{n} \rightarrow 0$. In the second case, $\left(1 / s_{n}\right)\left(s_{n} x\right) \rightarrow 0$, so $x=0$ which is a contradiction. In the first case, there are subsequences of $t_{n}$ which converge to $t$ and $t^{\prime}$ respectively so $t x=t^{\prime} x$. Hence if either $t_{n} \geqq 0$ or $t_{n} \leqq 0$ or if $x \neq-x$ then $t=t^{\prime}$ by Lemma 1.1. This is a contradiction so $t_{n}$ converges. It follows, in any case, that $m_{x}$ is a closed map on $R$ and therefore $R x$ is a closed subset of $E$. Also, $m_{x}$ is a homeomorphism on $P^{-}$and on $N^{--}$which is a homeomorphism on $R$ if and only if $x \neq-x$.

If $m_{x}$ is a homeomorphism on $R$ then $R x$ cuts $E$ since $R x$ is a closed subset of $E$. If $m_{x}$ is not a homeomorphism on $R$ then $x=-x$, $R x=P^{-} x$ and hence $R x$ does not cut $E$. The proof the theorem is complete.

1.3 Definition. If $x \neq 0$ then the ray through $x$, denoted $[x]$, is the set $P^{-} x$. The open ray through $x$, denoted $(x]$, is the set $P x$.

Evidently the union of any two distinct rays cuts $E$. In fact, if $[z]$ and $[w]$ are distinct rays and $T=[z] \cup[w]$ then $E \backslash T$ is the union of two disjoint (topological) planes which we are entitled to call sectors. For obviously if $[v]$ is a ray then $x \in(v]$ if and only if $(v]=(x]$, or equivalently, if and only if $(v] \cap(x] \neq \phi$. Thus if $S$ is one of the components of $E \backslash T$ and $x \in S$ then $(x] \subset S$.

1.4 Definition. An open sector is one of the components into which a pair of (not necessarily distinct) rays divides $E$, or the set $E \backslash\{0\}$. A sector denotes any one of the following sets:

(1) an open sector; 
(2) an open ray;

(3) the union of an open sector with one or both of its bounding open rays.

According to this definition, a sector never contains the origin.

Due to the commutativity of multiplication, the maps $m_{x}$ satisfy $m_{x}(t y)=t m_{x}(y)$ for all $t \in R, y \in E$. Hence if $m_{x}(y) \neq 0$ then $m_{x}((y])$ is an open ray. The squaring function $\chi$ (defined by the identity $\chi(x)=x^{2}$ for all $x \in E$ ) satisfies $\chi(t x)=t^{2} \chi(x)$ for all $t \in R, x \in E$. Hence if $E$ has no nilpotent elements, $\chi$ maps open rays onto open rays. A useful property of such functions is that they map sectors into sectors. In order to see this, observe that the set of open rays forms a decomposition $\mathscr{D}$ of $E \backslash\{0\}$. It is easy to see that in the quotient topology, $\mathscr{D}$ is homeomorphic to a circle and that under the quotient map $\nu$ the sectors simply correspond to connected subsets of $\mathscr{D}$. If $f$ is any map on a sector $S$ of $E$ which maps open says in $S$ onto open rays of $E$, then $f$ induces a map from $\nu(S)$ into $\mathscr{D}$. The image of $f$ is simply the inverse image, under $\nu$, of the image of some connected subset of $\mathscr{D}$, i.e., a sector. We record these facts for future reference in a form which is sufficiently general for our purposes.

1.5 Theorem. Suppose $S$ is a sector and $f: S \rightarrow E$ is a map such that (i) $x \in S$ implies $f(x) \neq 0$; (ii) $x \in S$, $t \in R$ implies there exists a positive integer $n$ such that $f(t r)=t^{n} f(x)$. Then $f(S)$ is a sector in $E$.

We recall next the well know fact that the set of fixed points of an involution on a circle $K$ must either be all of $K$, precisely two points, or empty. Our interest in involutions lies in the fact that if $x \in E$ and $x^{2}=1$, then $m_{x}$ is an involution on $E$. Furthermore, if, as above, $\mathscr{D}$ denotes the decomposition on $E-\{0\}$ into open rays, $m_{x}$ induces an involution on $\mathscr{D}$. It then follows easily that the set, $F\left(m_{x}\right)$ of fixed points (in $E$ ) of $m_{x}$ is either all of $E$, the union of two distinct rays or zero alone.

Which of the several possibilities obtain in a given instance can sometimes be settled in the following way: Suppose a pair of distinct open rays $(z]$ and $(w]$ are interchanged by $m_{x}$, i.e., $m_{x}((z])=(w]$. Let $S_{1}, S_{2}$ be the two sectors into which $[z] \cup[w]$ divides $E$. Observe that $m_{x}\left(S_{1} \cup S_{2}\right)=S_{1} \cup S_{2}$. If $S_{1}$ and $S_{2}$ are interchanged by $m_{x}$, i.e., $m_{x}\left(S_{1}\right)=S_{2}$, then only the origin is left fixed. If eithers $S_{1}$ or $S_{2}$ is mapped into itself by $m_{x}$, or in fact, if either $m_{x}\left(S_{1}\right) \cap S_{1}$ or $m_{x}\left(S_{2}\right) \cap S_{2}$ is non-empty, then $m_{x}\left(S_{1}\right)=S_{1}, m_{x}\left(S_{2}\right)=S_{2}$, and each of $S_{1}$ and $S_{2}$ contain an open ray of fixed points. In general, if $F\left(m_{x}\right)=[u] \cup[v]$, and if $S, S^{\prime}$ are the sectors into which $[u] \cup[v]$ divides $E$ then $S$ and $S^{\prime}$ are interchanged by $m_{x}$. 
These results are summarized in the following important theorem.

1.6 Theorem. Suppose $x \in E$ and $x^{2}=1$. Then either

(1) $F\left(m_{x}\right)=\{0\}$,

(2) $F\left(m_{x}\right)=[u] \cup[v]$ for some pair of distinct rays $[u]$ and $[v]$ and $m_{x}$ interchanges the two sectors into which $[u] \cup[v]$ divides $E$, or

(3) $F\left(m_{x}\right)=E$. In particular, suppose a pair of distinct rays [z] and [w] are interchanged by $m_{x}$ and let $S_{1}, S_{2}$ denote the sectors into which $[z] \cup[w]$ divides $E$. Then: $F\left(m_{x}\right)=\{0\}$ if and only if $S_{1}$ and $S_{2}$ are interchanged, while $F\left(m_{x}\right)=[u] \cup[v]$ for some $u \in S_{1}, v \in S_{2}$ if and only if either $m_{x}\left(S_{1}\right) \cap S_{1} \neq \phi$ or $m_{x}\left(S_{2}\right) \cap S_{2} \neq \phi$.

1.6.1 Corollary. Suppose $x \notin R$ and that $R x$ cuts $E$. If $R x$ does not separate 1 from -1 then each sector of $E \backslash R x$ contains an element $y$ such that $-y=y$.

Proof. Let $m(y)=-y$ for all $y \in E$. Let $S_{1}$ be the sector of $E \backslash R x$ which contains 1. Then $1 \in m\left(S_{1}\right) \cap S_{1}$ and the desired conclusion follows from the theorem.

1.6.2 CoRollary. Let $U, V$ denote the sectors of $E \backslash R$. Then either (1) $-U=V$ or (2) $-U=U$. Furthermore, condition (1) is equivalent to any one of the following conditions:

(3) $R$ separates $x$ and $-x$ for all $x \notin R$;

(4) $-x \neq x$ for any $x \neq 0$;

(5) $R$ separates $x$ and $-x$ for some $x \notin R$;

(6) There exists an $x$ such that $R x$ cuts $E$ and separates 1 and -1 ;

(7) Rx separates 1 and -1 for all $x \notin R$;

(8) for all $x \neq 0, R x$ cuts $E$ and separates $y$ and $-y$ if $y \notin R x$.

Condition (2) is equivalent to (9) there exists an element $u \in U$ and $v \in V$ such that $-u^{\prime}=u^{\prime}$ if $u^{\prime} \in[u]$ and $-v^{\prime}=v^{\prime}$ if $v^{\prime} \in[v]$.

Proof. Set $m(y)=-y$ for all $y \in E$. The various assertions follow directly from the theorem, the previous corollary or the fact that $R y$ separates $x$ and $-x$ if and only if $R x$ separates $-y$ and $y$.

2. In this section we study the shape and nature of $G$, the component of the identity in the set $H(1)$ of elements having inverses with respect to 1 . Recall that $L$ denotes the boundary of $G$ in $E$. According to [5], $G$ and $H(1)$ are Lie groups and hence are open subsets of $E$. Furthermore, $L$ is an ideal in $G \cup L$.

Evidently $0 \in L$. Suppose that $L$ contains a non-zero element $e$. Since $P^{-} \subset G^{-}, P^{-} e \subset L$. However, $P^{-} e \neq L$. For if $P^{-} e=L$ then a closed subset of $E$, homeomorphic to the ray $P^{-}$forms the boundary of 
the open set $G$. On purely topological grounds, $G=E \backslash P^{-} e=E \backslash L$. Thus $-e \notin G$ so $-e \in P^{-} e$, whence $-e=e$. According to the result of the previous section, whenever multiplication by -1 has one ray of fixed points, it has another. That is, there exists $u \notin[e]$ such that $-u=u$. Therefore $u \in G$. However, if $u \in G$ and $-u=u$ then $-1=1$, which is absurd. Hence $L \neq[e]$, and we have proved

\subsection{Theorem. The boundary $L$ of $G$ is not a ray.}

Continue to suppose $L$ contains a non-zero element $e$. If $L=R e$ (in which case $e \neq-e$ ), then $G \cup L$ is a semigroup on a half-plane with $G$ the open half plane. Therefore, by $[5, \S 4], G$ is isomorphic to the two dimensional vector group and 0 divides $L$ into two subsets $A$ and $B$ such that $A B=0$. The two sets $A$ and $B$ in this case must be $P e$ and Ne. Therefore $e^{2}=0$. That this case can occur is shown in Example 1 of the last section. However, it is impossible if $E$ has no nilpotent elements.

2.2 THEOREM. If $E$ contains no nilpotent elements and $L$ has more than one element then $L \neq R e$ for any $e \in L$.

If $L$ contains two distinct rays $\left[e_{1}\right],\left[e_{2}\right]$ (as it must, as we have just seen, if $E$ has no nilpotent elements) then $L=\left[e_{1}\right] \cup\left[e_{2}\right]$. For if there exists $e \in L \backslash\left(\left[e_{1}\right] \cup\left[e_{2}\right]\right)$ then $[e] \subset L,(e] \cap\left(\left[e_{1}\right] \cup\left[e_{2}\right]\right)=\phi$ and $[e]$ divides $G \cup L$ into two sectors. But then $G$ would be contained in one of these sectors. Thus if $\left[e_{i}\right]$ formed one of the bounding rays of $G$, no point (save zero) of $\left[e_{j}\right]$ could be boundary point of $G$, which is a contradiction (here, $i, j=1$ or 2 and $i \neq j$ ). Thus, if $L$ contains two distinct rays then it is the union of them. Observe that this is even true in case $L=R e$ (for $R e=[e] \cup[-e]$ ). Therefore, in either case, $G \cup L$ is a semigroup on a half-plane in which $G$ in the open half-plane. Therefore $G$ is isomorphic to the two dimensional vector group and 0 divides $L$ into two sets $A$ and $B$ so that $A B=0$ and either

(i) $A^{2}=B^{2}=0$,

(ii) $A$ and $B$ are groups, or

(iii) $A$ is a group and $B^{2}=0$. Evidently $A=P e_{1}$ if $\mathrm{e}_{1} \in A$ and $B=P e_{2}$ if $e_{2} \in B$. Thus we have the following

2.3 THEOREm. If $E$ is a real commutative semigroup and $L$ has more than one point then $L$ is the union of two distinct rays and $G$ is isomorphic to the two dimensional vector group. If $E$ contains no nilpotent elements then $L$ contains two non-zero idempotents $e_{1}, e_{2}$ and $L=\left[e_{1}\right] \cup\left[e_{2}\right]$. 
It is apparent that $L=\{0\}$ if and only if $H(1)=G$, i.e. if and only if $H(1)$ has only one component. If $G=H(1)$ then $G$ is not isomorphic to the two dimensional vector group. Therefore $G$ contains a non-trivial compact subgroup [6; Theorem 41]. Hence, by [4], $G$ is isomorphic to the product of the circle group and the multiplicative group of positive real numbers. It is then an easy matter to prove that $E$ is isomorphic to the multiplicative semigroup of complex numbers. This result also follows from [2] as well as [4].

2.4 THEOREM. If $E$ is a real commutative semigroup then the following conditions are equivalent:

(1) $E$ is isomorphic to the multiplicative semigroup of complex numbers;

(3) $L=\{0\}$ :

(3) $H(1)$ is connected.

3. The question was raised in [5] whether multiplication on $G$ and $L$ separately determines multiplication on $G \cup L$. We do not answer this question in general, but show that, under our hypotheses, if $L$ contains two non-zero idempotents then there is only one semigroup on $G \cup L$. In particular, $G \cup L$ is isomorphic to the product semigroup on $P^{-} \times P^{-}$.

For $e \in L$, let $G_{e}=\{g \in G: g e=e\} ; G_{e}$ is the isotropy group of $e$ in $G$. If $e \neq 0$ then $G_{e}$ is isomorphic to the multiplicative group of positive real numbers. For $\operatorname{dim} G_{e}=\operatorname{dim} G-\operatorname{dim}(G e)=\operatorname{dim} G-\operatorname{dim}(e]=1$. Furthermore, $G_{e}$ is a closed subset of $G$ and $G$ has no non-trivial compact subgroups. Since $G e=R e, G e$ is not compact. These conditions imply that $G_{e}$ is isomorphic to the multiplicative group of positive real numbers. (This proof is patterned after the proof of 3.6 of [5]).

3.1 Lemma. Let $e \in L, e \neq 0$. Then $e^{2}=e$ if and only if $e \in G_{e}^{-}$.

Proof. In general, if $x \in G_{e}^{-}$then $e=e x$ so if $e \in G_{e}^{-}$then $e^{2}=e$.

Conversely, suppose $e^{2}=e$. Then $P \neq G_{e}$ since $0 \in P^{-}$and $e \neq 0$. If $R_{1}$ and $R_{2}$ are any one-parameter subgroups of $G, G=R_{1} \cdot R_{2}$. Therefore $G=P \cdot G_{2}$. Since $e \in G^{-}$there is a sequence $t_{n}$ in $P$ and a sequence $g_{n} \in G_{e}$ such that $t_{n} g_{n} \rightarrow e$. Therefore $t_{n} g_{n} e \rightarrow e$ so $t_{n} e \rightarrow e$. Thus $t_{n} \rightarrow 1$ so $\left(1 / t_{n}\right)\left(t_{n} g_{n}\right) \rightarrow e$. Hence $g_{n} \rightarrow e$ and $e \in G_{e}^{-}$.

We are indebted to the referee for suggestions which lead to a considerable shortening of our proof of the following theorem.

3.2 THEoREm. If $L$ contains two non-zero idempotents then $G \cup L$ is isomorphic to $P^{-} \times P^{-}$.

Proof. Let $e_{1}, e_{2}$ denote the non-zero idempotents of $L$ and let $G_{i}$ 
denote the isotropy group of $e_{i}, i=1,2$. By the lemma, $G_{1} \neq G_{2}$ for then $e_{1} e_{2}=e_{2}$ and $e_{2} e_{1}=e_{1}$. Thus $G=G_{1} G_{2}$ and hence $G e_{1}=G_{2} e_{1}$ and $G e_{2}=G_{1} e_{2}$. Moreover, $G_{1}^{-}=G_{1} \cup\left\{e_{1}\right\}$ and $G_{2}^{-}=G_{2} \cup\left\{e_{2}\right\}$ and the map from $G_{1}^{-} \times G_{2}^{-}$to $G \cup L$ defined by $(x, y) \rightarrow x y$ is one-to-one and onto (clearly so on $G_{1} \times G_{2}$, on $e_{1} \times G_{2}$, on $G_{1} \times e_{2}$ and on $\left\{e_{1}, e_{2}\right\}$ independently and hence everywhere). Further, if $K$ is a compact subset of $G \cup L$ then $K e_{1}$ and $K e_{2}$ are compact subsets of $P^{-} e_{1}$ and $P^{-} e_{2}$ respectively. Therefore there are elements $g \in G_{1}, h \in G_{2}$ such that $k e_{1} \leqq h e_{1}$ and $k e_{2} \leqq g h_{1}$ for all $k \in K\left(P^{-} e_{1}\right.$ and $P^{-} e_{2}$ are ordered in the natural way). If we let $\left[e_{1}, g\right]$ denote the subset of $G_{1}^{-}$between $e_{1}$ and $g$ (and similarly for $\left.\left[e_{2}, h\right]\right)$, it follows that $K \subset\left[e_{1}, g\right] \cdot\left[e_{2}, h\right]$. Now our mapping sends $\left[e_{1}, g\right] \times\left[e_{2}, h\right]$ onto $\left[e_{1}, g\right] \cdot\left[e_{2}, h\right]$ and is a homeomorphism there. Thus $K$ comes from a compact subset of $G_{1}^{-} \times G_{2}^{-}$. Hence, the mapping $(x, y) \rightarrow x y$ is a homeomorphism. Since $P^{-}, G_{1}^{-}$and $G_{2}^{-}$are isomorphic, the theorem follows.

As a corollary to this theorem and Theorem 2.3 we have

3.2.1 CoRollary. If $E$ is a real commutative semigroup without nilpotent elements and $L$ has more than one element then $G \cup L$ is isomorphic to the product semigroup on $P^{-} \times P^{-}$.

4. If $C$ is a component of $H(1)$ then $C=x G$ for $x \in C$, so that $C$ is an open subset of $E$. Therefore $H(1)$ has at most a countable number of components. In this section we show that if $E$ has no nilpotent elements then $H(1)$ has only a finite number of components, in fact, a maximum of four.

Suppose $H(1)$ has an infinite number of components $C_{1}, C_{2}, \cdots, C_{n} \cdots$. Choose $x_{i} \in C_{i}$ for each $i$. Let $e_{1}, e_{2}$ be elements lying in distinct open rays of $L$. Thus $C_{i}=x_{i} G$ and each $C_{i}$ is a sector with bounding rays $\left(x_{i} e_{1}\right]$ and $\left(x_{i} e_{2}\right]$. Let $K$ be a (topological) circle with the origin on the inside. Then each of the rays $\left(x_{i} e_{1}\right]$ intersects $K$ in at least one point. Since a given ray $\left(x_{i} e_{1}\right]$ can form the common boundary of at most two of the components of $H(1)$, and since two open rays either identical or disjoint, it is possible to choose an infinite number of points $s_{n}$ in $K$ so that each $s_{n}$ belongs to some open ray $\left(x_{i} e_{1}\right]$ and so that no two of the $s_{n}$ belong to the some open ray. This set of points must have a cluster point $k \in K$. At most one of the $s_{n}$ can belong to $(k]$. Assume $s_{1} \notin(k]$. Then one of the two sectors of $E \backslash[k] \cup\left[s_{1}\right]$ contains infinitely many of the $s_{n}$. Call that one $S_{1}$; it does no harm to assume all of the $s_{n}$ belong to $S_{1}$. The $s_{n}$ can be re-numbered so that for each $n>1, s_{n+1}$ belongs to the sector $S_{n}$ of $E \backslash[k] \cup\left[s_{n}\right]$ which does not contain $s_{1}$. Therefore $\left(s_{n+1}\right] \subset S_{n}$.

If $U$ is a Euclidean neighborhood of $k$ which contains $s_{n}$ and $s_{n+1}$, for example, every open ray which is contained in $S_{n}$ must 
intersect $U$. Therefore if $s_{n+1} \in\left(x_{j} e_{1}\right]$ then $\left(x, e_{2}\right] \cap U \neq \phi$. Thus it is possible to choose a sequence of points $t_{n} \in E e_{2}$ so that $t_{n} \rightarrow k$, while $s_{n} t_{n}=0$ for every $n$. Hence $k^{2}=0$, so if $E$ has no nilpotent elements then $H(1)$ has only a finite number of components.

Assume that $E$ has no nilpotent elements. Set $G=C_{0}$, and let the remaining components be denoted $C_{1}, \cdots, C_{n}$. When $L$ has more than one element, $n \neq 0$. According to Example 2 of $\S 6, n=1$ is possible. Assume that $n>1$. Since $(-G)(-G)=G, H(1) / G$ has an element of order 2 . Therefore $H(1)$ has an even number of components, so $n \geqq 3$. We show that $n=3$ and that $H(1) / G$ is the four group.

First, order the rays that lie in the complement of $G$.

4.1 Definition. If $x, y \notin G^{-}$and if $(x]$ and $(y]$ are distinct, then $(x]<(y]$ provided $x$ and therefore $(x])$ is contained in the sector of $E \backslash\left[e_{2}\right] \cup[y]$ which does not contain $G$. For any $x \notin G^{-},\left(e_{2}\right]<(x]<\left(e_{1}\right]$. If $S_{1}$ and $S_{2}$ are disjoint sectors contained in the complement of $G$ then $S_{1}<S_{2}$ if for every $x \in S_{1}$ and $y \in S_{2},(x]<(y]$.

The collection of sectors $C_{1}, \cdots, C_{n}$ forms a linearly ordered collection of sectors according to this definition. Assume that they are numbered so that $C_{1}<C_{2}<\cdots<C_{n}$. Let $D_{i}$ denote the sector between $C_{i-1}$ and $C_{i}$ for $i=1, \cdots, n$ and let $D_{n+1}$ denote the sector between $C_{n}$ and $\left(e_{1}\right]$. The sectors $D_{i}, i=1, \cdots, n+1$, are to include their bounding open rays.

Let $\chi$ denote the squaring function: $\chi(x)=x^{2}$. Since $E$ has no nilpotent elements, $\chi$ maps sectors into sectors, according to Theorem 1.3. In addition, $\chi$ has the following special properties:

(1) $\chi\left(\bigcup_{i=0}^{n} c_{i}\right)=\bigcup_{i=0}^{n} C_{i}$;

(2) $\chi\left(\bigcup_{i=1}^{n+1} D_{i}\right) \subset \bigcup_{i=1}^{n+1} D_{i}$;

(3) $\chi\left(C_{0}\right)=C_{0}$ and $\chi\left(C_{i}\right) \cap C_{i}=\phi$ if $i>0$. The latter part of (3) is due to the fact that $C_{i}=x_{i} G$ for $x_{i} \in C_{i}$. Therefore $\chi\left(C_{i}\right)=x_{i}^{2} G$. But if $x_{i}^{2} \in C_{i}$ then $x_{i}^{2}=x_{i} g$ for some $g \in G$, so $x_{i} \in G$ which is a contradiction.

If $x$ is sufficiently near $e_{3}$ then $x^{2}$ is near $e_{2}$. Therefore $\chi\left(D_{1}\right) \subset D_{1}$. Furthermore, $\chi\left(C_{1}\right)$ is a sector having boundary points in common with $\chi\left(D_{1}\right)$, and $\chi\left(C_{1}\right)$ is disjoint from $C_{1} \cup D_{1}$. Thus $\chi\left(C_{1}\right)$ must have points in common with $G$, whence $\chi\left(C_{1}\right)=G$. It follows that $C_{1}$ contains an element whose square is 1 . Hereafter, $x_{1}$ will denote this element; i.e., $x_{1} \in C_{1}$ and $x_{1}^{2}=1$.

Choose $x_{i} \in C_{i}$ for $i=2, \cdots, n$. Now

$$
x_{1}\left(C_{1} \cup C_{2}\right)=x_{1}\left(x_{1} G \cup x_{2} G\right)=G \cup x_{1} x_{2} G .
$$

Set $S=C_{1} \cup D_{2} \cup C_{2}$. Then $x_{1} S$ is a sector which contains $G \cup x_{1} x_{2} G$ and no other components of $H(1)$. Therefore either $x_{1} x_{2} G=x_{1} G$ or $x_{1} x_{2} G=x_{n} G$. But $x_{1} x_{2} G=x_{1} G$ implies $x_{2} G=G$ which is a contradiction. Therefore $x_{1} x_{2} G=x_{n} G$. On the other hand, $x_{2}\left(x_{1} G \cup G\right)=x_{2} x_{1} G \cup x_{2} G$. 
An argument similar to the preceding shows that $x_{2} x_{1} G$ is either $x_{1} G$ or $x_{3} G$. Since $x_{2} x_{1}=x_{1} x_{2}, x_{2} x_{1} G \neq x_{1} G$. Therefore $x_{2} x_{1} G=x_{3} G$. But

$$
x_{2} x_{1} G=x_{1} x_{2} G=x_{n} G .
$$

Hence $n=3$, so $H(1)$ has four components.

The argument used above to show that $C_{1}$ contains an element whose square is 1 applies with only trivial changes to $C_{3}$. Therefore we assume that $x_{3}^{2}=1$. Thus $H(1) / G$ has four elements, at least two of which have order two. Therefore $H(1) / G$ is the four group and $\left(C_{i}\right)\left(C_{i}\right)=G$ for $i=0,1,2$, or 3 . We have proved the following result.

4.2 THEoRem. If $E$ is a commutative real semigroup without nilpotent elements then $H(1)$ has one, two or four components. When $H(1)$ has four components, $H(1) / G$ is the four group.

5. Throughout this section $E$ is assumed to be a real commutative semigroup without nilpotent elements. Thus if $L$ has more than one element, as it will if $H(1)$ has more than one component, then $L$ contains two non-zero idempotents and $G \cup L$ is isomorphic to $P^{-} \times P^{-}$.

Even if $H(1)$ has four components a question remains concering the location of $-G$. In a certain sense the question is irrelevant and $-G$ can be any one of $C_{1}, C_{2}$ or $C_{3}$; in this connection see Remark 5.4. However, the "correct" location for $-G$, viz. $-G=C_{2}$, is recognizable, modulo the choice of $R$.

5.1 Theorem. Assume that $E$ has no nilpotent elements, and that $L$ has more than one element. Then precisely one component of $H(1)$ lies between $G$ and $-G$ if and only if $R$ separates $x$ and $-x$ for some $x$ (equivalently, $x \neq 0$ implies $x \neq x$ ). In particular, if $x \neq 0$ implies $x \neq-x$ then $H(1)$ has four components.

Proof. Let $e_{1}$ and $e_{2}$ denote the non-zero idempotents of $L$. If $x \neq-x$ for $x \neq 0$ then $\left(-e_{1}\right]<\left(-e_{2}\right]$ (in the sense of Definition 4.1). Let $S$ denote the open second quadrant-that is the sector of elements $x$ such that $\left(e_{2}\right]<(x]<\left(-e_{1}\right]$. Thus $E=G \cup(-G) \cup\left(S^{-}\right) \cup\left(-S^{-}\right)$. Let $C=\left\{x \in S: x^{2} \in S^{-}\right\}$and $D=\left\{x \in S: x^{2} \in-S^{-}\right\}$. If $S \cap H(1)=\phi$ then $S=C \cup D$. Since $C$ and $D$ are closed subsets of $S$ and $S$ is connected, if both $C$ and $D$ are non-empty then $C \cap D \neq \phi$. But if $x \in C \cap D$ then $x^{2} \in S^{-} \cap\left(-S^{-}\right)$so $x^{2}=0$ which is a contradiction. Therefore either $S=C$ or $S=D$. If $x$ is sufficiently near $e_{2}$ then $x^{2} \notin-S^{-}$while if $x$ is sufficient near $-e_{1}$, then $x^{2} \notin S^{-}$. Therefore $H(1) \cap S \neq \phi$ so that at leat one component of $H(1)$ is contained in $S$-that is to say, between $G$ and $-G$. A similar argument shows that one component of $H(1)$ is 
contained in $-S$. Since $H(1)$ can have at most four components it must in this case have exactly four. Therefore precisely one component of $H(1)$ lies between $G$ and $-G$.

Conversely, suppose precisely one component of $H(1)$ lies between $G$ and $-G$; i.e., $-G=C_{2}$. Now $-C_{1} \neq G$ and $-C_{1} \neq-G$ so $-C_{1}=C_{3}$. Therefore there exists $x \in E$ which is separated from $-x$ by $R$. The proof of the theorem is complete.

Under the hypotheses of the previous theorem the relative positions of $-e_{1}, x_{1}$ and $-e_{2}$ are known and according to this theorem $\left(x_{1}\right]<\left(-e_{1}\right]$. (Recall that $x_{1}$ is the member of $\mathrm{C}_{1}$ such that $x_{1}^{2}=1$ ). However, which of $\left(x_{1} e_{1}\right]$ and $\left(x_{1} e_{2}\right]$ occurs first has not yet been determined. Since $x_{1} G$ forms the component of $E \backslash\left[x_{1} e_{1}\right] \cup\left[x_{1} e_{2}\right]$ which does not contain $G,\left(x_{1}\right]$ is contained between $\left(x_{1} e_{1}\right]$ and $\left(x_{1} e_{2}\right]$. Let $e$ be the member of the pair $\left\{e_{1}, e_{2}\right\}$ such that $\left(x_{1} e\right]<\left(x_{1}\right]$. Let $S$ denote the sector of $E-\left[x_{1} e\right] \cup\left(e_{1}\right]$ which contains $G$. Let $A$ be an arc from $e_{2}$ to $x_{1}$ which is contained entirely in $S \cup\left\{e_{2}\right\}$. Then $e_{2} A$ is connected and cannot contain any elements of $H(1) \cup\{0\}$. Since $e_{2} \in e_{2} A, e_{2} A \subset D_{1}$ so $\left(x_{1} e_{2}\right]<\left(x_{1}\right]$ and $e=e_{2}$.

The two rays $\left(e_{1}\right]$ and $\left(x_{1} e_{1}\right]$ are distinct and are interchanged under multiplication by $x_{1}$. Since $x_{1}\left(x_{1} G\right)=G$, each of the sectors of $E \backslash\left[e_{1}\right] \cup\left[x_{1} e_{1}\right]$ is mapped into itself by $x_{1}$ and therefore each of these sectors contains a ray of fixed points of $x_{1}$. Therefore there exists a (non-zero) point $z \in D_{1}$ such that if $y \in D_{1}$ then $x_{1} y=y$ if and only if $y \in[z]$. Either $\left(x_{1} e_{2}\right]=D_{1}=\left(e_{2}\right]$ and $x_{1} e_{2}=e_{2}$, or $z$ is in the interior or $D_{1}$. Now in the proof preceding Theorem 4.2 (which only used hypotheses available here) it was shown that if $x \in D_{1}$ then $x^{2} \in D_{1}$. Now $x_{1} z^{2}=$ $\left(x_{1} z\right) z=z^{2}$ so $z^{2} \in(z]$. Therefore $z^{2}=t z$ for some $t>0$ so $z / t$ is an idempotent in $D_{1}$ which is different from $e_{2}$ unless $x_{1} e_{2}=e_{2}$, and which is different from zero in any case. In a similar fashion it can be shown that $D_{4}$ contains a non-zero idempotent which is different from $e_{1}$ unless $x_{3} e_{1}=e_{1}$. (Where $x_{3} \in C_{3}$ and $\left.x_{3}^{2}=1\right)$.

Therefore, if $E$ has no nilpotent element, $x \neq 0$ implies $x \neq-x$ and if $E$ has exactly four idempotents then $x_{1} e_{2}=e_{2}$ and $x_{3} e_{1}=e_{1}$. Furthermore, since $-x_{1}=x_{3}$, we have $x_{3} e_{2}=-e_{2}$ and $x_{1} e_{1}=-x_{1}$. Thus

$$
E=G^{-} \cup\left(-G^{-}\right) \cup C_{1} \cup C_{3}
$$

and it is now an easy matter to construct an isomorphism from $E$ onto $R \times R$. (For this purpose, make use of Corollary 3.2.1).

There are several alternatives to the hypothesis that $E$ contains exactly four idempotents. For if $L$ contains more than one element and it is assumed that either $H(1)$ is dense in $E$ or $L \cup(-L)$ is an ideal in $E$ then $x_{1} e_{2}=e_{2}, x_{1} e_{1}=-e_{1}$, etc. and the conclusion that $E$ is isomorphic to $R \times R$ follows as before. In virtue of the existence of non-zero idempotents in $D_{1}$ and $D_{4}$, another alternative is to assume that $x^{2} \in G^{-}$ 
for all $x \in E$. Finally, since $\left(x_{1} e_{2}\right)^{2}=e_{2},\left(x_{1} e_{1}\right)^{2}=e_{1}$, etc., it may be assumed that $x \notin L \cup-L$ implies $x^{2} \notin L \cup(-L)$. We have proved the following result.

5.2 THEOREM. If $E$ is a real commutative semigroup without nilpotent elements and if $x \neq 0$ implies $x \neq-x$ then the following conditions are equivalent:

(1) $E$ is isomorphic to $R \times R$;

(2) $E$ has precisely four idempotents;

(3) $L$ contains more than one element and $H(1)$ is dense in $E$;

(4) $L$ contains more than one element and $x \in E$ implies $x^{2} \in G^{-}$;

(5) $L$ contains more than one element and $L \cup(-L)$ is an ideal in $E$;

(6) $L$ contains more than one element and $x \notin L \cup(-L)$ implies $x^{2} \notin L \cup(-L)$.

The examples in $\S 6$ show that none of the conditions (2)-(6) of the previous theorem implies $E$ is isomorphic to $R \times R$ if the condition that $x \neq 0$ implies $x \neq-x$ is simply dropped. However, a certain weakening of this condition is possible.

Our proof of this fact makes use of Theorem 5.2.

5.3 THEOREM. If $E$ is a real commutative semigroup without nilpotent elements and if $x \in L \backslash\{0\}$ implies $x \neq-x$ then conditions (1)-(4) of the previous theorem are equivalent.

Proof. Suppose $E$ contains precisely four idempotents. Then $L$ contains two non-zero idempotents $e_{1}$ and $e_{2}$. By hypothesis, $-e_{1} \neq e_{1}$ and $-e_{2} \neq e_{2}$. If $\left(-e_{1}\right]<\left(-e_{2}\right]$ then all of the hypotheses of the previous theorem are satisfied so $E$ is isomorphic to $R \times R$.

Therefore suppose $\left(-e_{2}\right]<\left(-e_{1}\right]$. By theorem 5.1, either the sector between $\left(e_{2}\right]$ and $\left(-e_{2}\right]$ or the sector between $\left(-e_{1}\right]$ and $\left(e_{1}\right]$ is devoid of members of $H(1)$. There is little to distinguish between the two cases so we suppose the former holds; that is, $D_{1} \cap H(1)=\phi$. Since the rays $\left(-e_{1}\right]$ and $\left(e_{1}\right]$ are interchanged under multiplication by -1 and $-(-G)=G$, there is a (non-zero)point $z \in D_{1}$ such that if $y \in D_{1}$, then $-y=y$ if and only if $y \in[z]$. As we have seen before, $z^{2} \in D_{1}$, so $D_{1}$ contains a nonzero idempotent; this idempotent must be distinct from $e_{2}$ since $-e_{2}=e_{2}$. But this means $E$ has a fifth idempotent, which is a contradiction. Hence $\left(-e_{1}\right]<\left(-e_{2}\right]$ and $(2)$ implies (1).

If $L$ contains more than one element then $E$ has at least four idempotents. If $E$ contains five idempotents then there is an idempotent $e \notin G^{-} \cup\left(-G^{-}\right)$. Therefore $P^{-} e$ and $R e_{1} \cup R e_{2}$ divide $E$ into five components whose union contains $H(1)$. But $H(1)$ can have at most four 
component so $H(1)$ is not dense in $E$. Therefore (3) implies (2).

Assume that (4) holds. Then $G^{-}$has four idempotents so since $x \in E$ implies $x^{2} \in G^{-}$there are no more. Therefore (4) implies (2). The remaining implications are obvious so the proof of the theorem is complete.

It is to be noticed that conditions (5) and (6) of Theorem 5.2 are not listed in Theorem 5.3. Example 4 of $\$ 6$ shows that (6) cannot be included while Example 5 shows that Condition (4) cannot.

5.4 REMARK. The previous two theorems yield criteria on $E$ in order that it be isomorphic to $R \times R$ under a sort of canonical isomorphismnamely an isomorphism in which $R$ maps onto the diagonal of $R \times R$. Conditions that there exist some isomorphism can be made somewhat weaker. Assume that $H(1)$ is known to have four components $G, C_{1}, C_{2}$ and $C_{3}$ where, as usual, $C_{1}<C_{2}<C_{3}$. Which one of these last three components is $-G$ depends on the particular choice of the isomorph of the real numbers in the beginning. For $-G$ is simply the component of $H(1)$ containing -1 . Set $R_{i}=\left(P x_{i}\right) \cup P^{-}$where $x_{i} \in C_{i}$ and $x_{i}^{2}=1$. Obviously $R_{i}$ is a sub-semigroup of $E$ which is isomorphic to $R$. Therefore by replacing a given choice of $R$ by another (isomorphic) choice, it becomes apparent that every such semigroup on $E$ in which $-G$ is a given $C_{i}$ is isomorphic (under the identity isomorphism) to one in which $-G$ is $C_{j}, i, j=1,2$ or 3 .

5.5 THEOREM. If $E$ is a real commutative semigroup without nilpotent elements in which for at least one $x \in L,-x \neq x$ then the following conditions are equivalent:

(1) $E$ is isomorphic to $R \times R$;

(2) $E$ has precisely four idempotents;

(3) $H(1)$ is dense in $E$;

(4) $x \in E$ implies $x^{2} \in G^{-}$.

Proof. The hypotheses imply that $L$ has two non-zero idempotents $e_{1}$ and $e_{2}$ and we may suppose $-e_{1} \neq e_{1}$. If $-e_{2} \neq e_{2}$ then the hypotheses of Theorem 5.3 are satisfied and the conditions are already known to be equivalent.

Suppose $e_{2}=-e_{2}$ and let $S$ be the component of $E \backslash R e_{1}$ which does not contain $G$. There is $x \in S$ such that $-x=x$. Therefore $-x^{2}=x^{2}$ so $x^{2} \in\left(e_{2}\right] \cup(x]$.

If $x^{2} \in(x]$ then $S$ contains an idempotent so (2) cannot hold. If (4) holds then $x^{2} \in G^{-}$so $x^{2} \in\left(e_{2}\right]$. Thus, if either (2) or (4) holds then $x^{2} \in\left(e_{2}\right]$.

Therefore suppose $x^{2} \in\left(e_{2}\right]$. Let $C$ denote the sector of $E \backslash\left([x] \cup\left[e_{1}\right]\right)$ which does not contain $G$. An argument analogous to that given in the proof of Theorem 5.1 shows that there is $y \in C$ such that $y^{2} \in G \cup(-G)$. It follows that $H(1)$ has a third, and therefore a fourth, component. 
Under the present circumstances, these components can be labeled $G$, $-G, C_{2}$ and $C_{3}$. Let $x_{2}$ be the member of $C_{2}$ such that $x_{2}^{2}=1$. Set $R_{2}=P^{-} \cup\left(P x_{2}\right)$. Replacing $R$ by $R_{2}$ yields a reals semigroup on $E$ which satisfies the hypotheses of Theorem 5.3. Conditions (2) and (4) are unaffected by this change. Therefore (2) implies (1) and (4) implies (1).

If $H(1)$ is dense then $H(1) \cap S \neq \phi$ so again we see that $H(1)$ has four components. Hence replacement of $R$ by $R_{2}$ as above yields a semigroup on $E$ satisfying the hypotheses of Theorem 5.3. Therefore (3) implies (1).

The remaining implications are obvious.

6. We conclude with several examples. In addition to being examples of the various classes of semigroups mentioned in the introduction, they reveal a certain amount of indepencence among the conditions in Theorem 5.2.

It is convenient, in one way or another, to regard $E$ as being coordinatized in the usual way. Thus we can speak readily of such terms as the $y$-axis, the open or closed first quadrant, etc.

All details concerning the proofs that the various multiplication are continuous and associative are omitted. However, many of the missing details are contained in the simple proof that the following canonical procedure for extending certain semigroups works: Suppose that $r$ is an involution on $E$ and that $S$ is a closed sector in $E$ so that $r(S) \cap S$ is either one of the bounding rays of $S$ or the origin, and that $r$ is the identity on $r(S) \cap S$. Assume that $S$ is endowed with the structure of a commutative semigroup so that $r(S) \cap S$ is a subsemigroup. Let $T=S \cup r(S)$. Define multiplication on $T$ as follows:

(1) if $x, y \in S$ then $x y$ has its original meaning;

(2) if $x \in S, y \in r(S)$ then $x y=y x=r(x r(y))$;

(3) if $x, y \in r(S)$ then $x y=r(x) r(y)$. Then $T$ is a commutative semigroup containing $S$ as a sub-semigroup. We shall refer to this semigroup as the extension of $S$ to $T$ by $r$.

All of the following examples satisfy condition (*).

EXAMPLE 1. Let $G \cup L$ be the semigroup "II b." of [5; p. 387] but regard it as embedded in the closed first quadrant (multiplication no longer has any simple relation to the coordinates). In other words, we have a commutative semigroup with identity on the closed first quadrant so that $G$ is the open first quadrant, $L$ is the union of the nonnegative $x$ and $y$-axes, $L^{2}=0$ and $G^{-}$has a sub-semigroup $P^{-}$which is isomorphic to the multiplicative semigroup of nonnegative real numbers, and $0 \in P^{-}$. Let $r(x, y)=(-y,-x)$ and let $S=G \cup L$. Then $T=$ $r(S) \cup S$ is the union of the first and third quadrants, and $r(S) \cap S=\{0\}$. 
Extend $S$ to $T$ by $r$. Next identify each point $(0,-y)$ with $(y, 0)$ and $(-x, 0)$ with $(0, x)$. The result is a semigroup on the plane which may be visualized as follows: $G$ is the upper half-plane, $-G$ is the lower half-plane, $R$ is the line $y=x$ and $L=R e$ where $e$ is a point on the positive $x$-axis.

In Example 1, every condition listed in Theorem 5.2 (except condition (1), of course) is satisfied except the condition on nilpotent elements; $H(1)$ has two components.

EXAMPLe 2. Let $G \cup L$ be the closed first quadrant with coordinatewise multiplication. Let $S=G \cup L$ and let $r$ be the involution given in Example 1. Let $T=S \cup r(S)$ and extend $S$ to $T$ by $r$. Identify points as in Example 1. The result can be pictured as a semigroup on a plane in which $G$ is the upper half-plane, $-G$ is the lower half-plane, $R$ is the line $y=x$ and $L=R e_{1} \cup R e_{2}$ where $e_{1}=(1,0)$ and $e_{2}=(-1,0)$. Both $e_{1}$ and $e_{2}$ are idempotents but $-e_{1}=e_{1}$ and $-e_{2}=e_{2}$.

In Example 2, all of the conditions listed in Theorem 5.2 (except Condition (1)) are met except the stipulation that $x \neq 0$ implies $-x \neq x$; $H(1)$ has two components.

EXAMPLE 3. Let $G \cup L$ be the closed first quadrant under coordinatewise multiplication. Let $P=\{(x, x): x>0\}$. Let $A$ be the arc of the unit circle which lies in the closed fourth quadrant. Let $e_{1}=(1,0)$ and $z=(0,-1)$. Assume that $A$ is endowed with the structure of a commutative semigroup so that $e_{1}$ is the identity and $z$ is a zero for $A$. Regard the closed fourth quadrant $Q$ as the product of $A$ and $P^{-}$(with all points $0 a, a \in A$, being identified with the origin). Let $S=G \cup L \cup Q$ and extend multiplication to $L$ as follows: if $g \in G \cup L, x \in Q$ then $g x=x g=\left(g e_{1}\right) x$. Observe that $\left(g e_{1}\right) x$ is well defined since $g e_{1} \in P^{-} e_{1}, e_{1}$ is the identity on $Q$ and multiplication of any $x \in Q$ and $t \in P^{-}$is the ordinary coordinate-wise product of $x$ and $t$. It is a simple matter to check that $S$ is a commutative semigroup containing $G \cup L$ as subsemigroup. Let $r$ be reflection about the $y$-axis. Then $E=S \cup r(S)$ is the plane and the extension of $S$ to $E$ by $r$ yields a real semigroup with the following properties:

(1) $E$ has no nilpotent elements;

(2) $H(1)$ has two components $G$ and $-G$ and $G^{-} \cap\left(-G^{-}\right)$is a ray;

(3) $H(1)$ is not dense in $E$.

ExAmPLE 4. Let $G \cup L$ be the closed first quadrant under coordinatewise multiplication. Let $P$ be as in Example 3 and let $e_{1}=(1,0)$, $e_{2}=(0,1)$. Let $r$ be the involution $r(x, y)=(-y,-x)$. Set $S=G \cup L$ and $T=S \cup r(S)$. Extend $S$ to $T$ by $r$. Thus $-e_{2}=(-1,0)$ and $-e_{1}=(0,-1)$. Let $Q_{1}$ and $Q_{2}$ denote the open second and fourth 
quadrants respectively. Let $A_{i}$ be the arc of the unit circle contained in $Q_{i}^{-}, i=1,2$. Let $z_{i} \in A_{i}$ be the point on the line $y=-x$ in $Q_{i}$. Assume that $A_{i}$ has the structure of a semigroup so that $e_{i}$ is an identity, $z_{i}$ is a zero and $\left(-e_{i}\right)^{2}=e_{i}, i=1,2$. Regard $Q_{i}^{-}$is the product of $A_{i}$ and $P^{-}$, indentifying all points $0 a, a \in A_{i}$, with the origin as usual. Extend multiplication to $E$ as follows: if $g \in G^{-}$and $x \in Q_{i}$ then $g x=$ $x g=\left(g e_{i}\right) x$ as in Example 3. Define multiplication by -1 to be reflection about the line $y=-x$ and if $g \in-G, x \in Q_{i}$, then

$$
g x=x g=-(-g x) .
$$

Finally, if $x \in Q_{1}, y \in Q_{2}$ define $x y=y x=0$. Then $E$ is a real semigroup with the following properties:

(1) $E$ has no nilpotent elements;

(2) $H(1)$ has two conponents $G$ and $-G$ and $G \cap\left(-G^{-}\right)=\{0\}$.

(3) $x \notin L \cup(-L)$ implies $x^{2} \notin L \cup(-L)$. This example shows that in the proof of Theorem 5.1 the condition that $R$ separate $e_{2}$ and $-e_{2}$ is essential.

ExAmple 5. Let $H$ denote the semigroup on the closed right halfplane defined as follows:

(1) $x y$ is the ordinary coordinate-wise product if either $x$ or $y$ belongs to the first quadrant; otherwise

(2) $x=\left(x_{1}, x_{2}\right), y=\left(y_{1}, y_{2}\right)$ with $x_{2} \leqq 0$ and $y_{2} \leqq 0$; define

$$
x y=\left(x_{1} y_{1},-\left(x_{2} y_{2}\right)\right) .
$$

Let $z$ be a point in the fourth quadrant on the line $y=-x$ and let $e_{2}=(0,1)$. Let $C$ denote the closed sector bounded by $[z]$ and $\left[e_{2}\right]$ which contains the first quadrant. Shrink the set $H$ to coincide with $C$ and let $S^{\prime}$ be the semigroup induced on $C$ by that on $H$. Let $r^{\prime}$ denote reflection about the line $y=-x$ and $T^{\prime}=S^{\prime} \cup r^{\prime}\left(S^{\prime}\right)$; extend $S^{\prime}$ to $T^{\prime}$ by $r^{\prime}$. Shrink $T^{\prime}$ to coincide with the closed right half-plane and let $S$ denote the induced semigroup. Finally, let $r$ be reflection about the $y$-axis. Let $T=S \cup r(S)$ and extend $S$ to $T$ by $r$. Choose $R$ to lie in the first and third quadrants. Then $E=T$ becomes a real semigroup with the following properties:

(1) $E$ has no nilpotent elements;

(2) $L$ contains more than one element and $x \in L \backslash\{0\}$ implies $x \neq-x$;

(3) $L \cup(-L)$ is an ideal in $E$. However, $E$ is not isomorphic to $R \times R$.

\section{REFERENCES}

1. W. M. Faucett, Compact semigroups irreducibly connected between two idempotents, Proc. Amer. Math. Soc., 6 (1955), 714-747. 
2. K. H. Hofmann, Lokalkompakte zusammenhängende topologische Halbgruppen mit dichter Untergruppe, Math. Ann., 140 (1960), 22-32.

3. J. G. Horne, Multiplications on the line, Proc. Amer. Math. Soc., 9 (1958), 791-795.

4. P. S. Mostert and A. L. Shields, On a class of semigroups on $E_{n}$, Proc. Amer. Math. Soc., 7 (1956), 729-734.

5. - Semigroups with identity on a manifold, Trans. Amer. Math. Soc., 91 (1959), 380-389.

6. L. Pontrjagin, Topological groups, Princeton University Press, 1946.

UNIVERSITY OF GEORGIA 
